\title{
Extended Quintessence with non-minimally coupled phantom scalar field
}

\author{
Orest Hrycyndf \\ Department of Theoretical Physics, Faculty of Philosophy, \\ The John Paul II Catholic University of Lublin, Al. Ractawickie 14, 20-950 Lublin, Poland \\ Marek Szydłowsk拧 \\ Astronomical Observatory, Jagiellonian University, Orla 171, 30-244 Kraków, Poland and \\ Mark Kac Complex Systems Research Centre, Jagiellonian University, Reymonta 4, 30-059 Kraków, Poland
}

\begin{abstract}
We investigate evolutional paths of an extended quintessence with a non-minimally coupled phantom scalar field $\psi$ to the Ricci curvature. The dynamical system methods are used to investigate typical regimes of dynamics at the late time. We demonstrate that there are two generic types of evolutional scenarios which approach the attractor (a focus or a node type critical point) in the phase space: the quasi-oscillatory and monotonic trajectories approach to the attractor which represents the FRW model with the cosmological constant. We demonstrate that dynamical system admits invariant two-dimensional submanifold and discussion that which cosmological scenario is realized depends on behavior of the system on the phase plane $\left(\psi, \psi^{\prime}\right)$. We formulate simple conditions on the value of coupling constant $\xi$ for which trajectories tend to the focus in the phase plane and hence damping oscillations around the mysterious value $w=-1$. We describe this condition in terms of slow-roll parameters calculated at the critical point. We discover that the generic trajectories in the focus-attractor scenario come from the unstable node. It is also investigated the exact form of the parametrization of the equation of state parameter $w(z)$ (directly determined from dynamics) which assumes a different form for both scenarios.
\end{abstract}

PACS numbers: 98.80.Bp, 98.80.Cq, 11.15.Ex

\section{INTRODUCTION}

The non-minimal coupling of the type $\frac{1}{2} \xi R \psi^{2}$ in the Lagrangian, where $R$ is the Ricci scalar and $\xi$ - the parameter of non-minimal coupling was independently introduced by Chernikov and Tagirov [1] and Callan et al. [2]. It was pointed out that we should consider $\xi \neq 0$ and different arguments were given. The nonzero $\xi$ arises from quantum corrections [3] and it is required by the renormalization [2]. Faraoni [4] argued that the non-minimal coupling of the inflaton field is unavoidable and in his opinion the paradigm of standard inflation should be generalized by including the coupling term in the Lagrangian. If the parameter $\xi$ assumes a value different from $1 / 6$ (conformal coupling case) then the equation of motion for the scalar field (i.e., the Klein-Gordon equation) is conformally invariant if $V(\psi)=0$ or $V(\psi) \propto \psi^{4}[5]$. While there are strong arguments that seems to favor the choice of $\xi=1 / 6$, under the assumption of conformal invariance of the Klein-Gordon equation and that $\psi$ does not violate the equivalence principle [6, 7] (contrary to Lightman et al. [8, p. 85]). Moreover the value of $\xi=1 / 6$ cannot be treated as a correct coupling for various scalar field particles. Adopting a different point of view [9] it is desirable to study different constraints on the coupling constant. The strength of the non-minimal coupling is constrained to be $\xi>-7.0 * 10^{-3}$ for negative $\xi$ in the case of a quadratic potential function [10].

Recent astronomical observations (like SNIa, the CMB and other [11, 12, 13, 14]) indicate that current universe is almost flat and undergoing accelerated phase of expansion. While the cosmological constant term introduced to the equations of general relativity offers the possibility of explanation of acceleration, its value is extraordinary small compared with an inferred vacuum energy. To solve this problem it is natural to consider a source of this acceleration in the form of a scalar field (quintessence idea 15, 16]). In the simplest case minimally coupled to gravity scalar fields with a potential $V(\psi)$ are considered. In this case the dynamical effect of a scalar field is equivalent to a perfect fluid with the energy density and the pressure given by $\rho_{\psi}=\frac{1}{2} \dot{\psi}^{2}+V(\psi)$ and $p_{\psi}=\frac{1}{2} \dot{\psi}^{2}-V(\psi)$, respectively. In the canonical quintessence scenario the kinetic energy is $k=\dot{\psi}^{2} / 2$ and the true cosmological constant is zero and the potential (tracking potential) is slow rolling down [17].

This quintessence field generically has good-or-bad attractor properties [18, 19], i.e., it is sensitive with respect

*Electronic address: hrycyna@kul.lublin.pl

†Electronic address: uoszydlo@cyf-kr.edu.pl 
to choice of the initial conditions. The tracking quintessence was invoked to use this attractor property to explain the smallness of the cosmological constant without fine tuning of the potential or initial conditions. The idea of quintessence was extended to the case of non-minimally coupled scalar field [20, 21, 22]. The main motivation of this extension was to explain the observational data which favor the negative equation of state parameter $w=p / \rho<-1$ [23]. If we consider the canonical scalar field in the background of Einstein gravity then we cannot explain the range of value $w<-1$. If we assume the flat universe then $w<-1$ implies superacceleration as a opposed to acceleration $\ddot{a} / a=\dot{H}+H^{2}>0$, where $a$ is the scale factor and $H=(\ln (a))^{\circ}$ is the Hubble parameter. There are two natural possibilities of extension of the theory: 1) to allow the scalar field $\psi$ to couple non-minimally to the Ricci scalar [24], and 2) including of the phantom scalar fields, i.e., the scalar field is formally allowed to have a negative kinetic energy and $\dot{\psi}^{2}$ is replaced by $(-) \dot{\psi}^{2}[25,26]$. This type of modification has a justification from string, $\mathrm{M}$ theory and in supergravity. Note that such possibilities can explain the breaking $w<-1$ barrier. Both phantom and non-minimally coupled scalar field models can be regarded as a scalar tensor theories of gravity. Such models are called extended quintessence [24, 27, 28]. Recently Fuzfa and Alimi suggested a promising unified description of both dark matter and dark energy. It relies on a violation of the weak equivalence principle on cosmological scales by dark matter. It is interesting that amplitude of this violation depends on the relative concentration of baryons [29].

The main aim of this paper is to investigate the long time evolution of extended quintessence models. We give the complete qualitative classification of solutions according to critical point approach scenarios (the monotonic approach to a critical point or the quasi-oscillatory regime of approaching and crossing of the $w=-1$ barrier). We use the dynamical system methods in investigation of all evolutional paths for all admissible initial conditions. These methods were previously used (see for example [30, 31]) in the context of the FRW model with non-minimally coupled scalar fields and superacceleration. We are interested in typical evolutional scenarios of the phantom non-minimally coupled scalar field at a late time near the $w=-1$ state. We demonstrate that there are two different scenarios to achieve this state. The new scenario of the route to the cosmological constant is through the damping oscillations of the scalar field asymptotically going to the constant value. In this scenario there is an infinite number of crossing the phantom divide line $w=-1$. In our previous paper [32] we study how deSitter state can be achieved in cosmology with conformally coupled to gravity phantom scalar field. The dynamics of the phantom scalar field conformally coupled to gravity with quadratic potential function depends on the value of squared mass of the field. The dynamics is regular for $m^{2}>0$, in opposite case with $m^{2}<0$ the dynamics becomes chaotic and fractals structure appears in the phase space [33]. In the present paper we generalize result of [32] by consideration $\xi$ as a free parameter which should be constrained by observational data. In investigation of dynamics with the help of dynamical system methods this parameter plays the role of bifurcation parameter and we found some critical value of this parameter for which topological structure of phase space changes. We show that damping oscillations around $w=-1$ value type of evolution can be described by a critical point of a focus type on a 2-dimensional invariant submanifold $\left(\psi, \psi^{\prime}\right)$ where ' $\equiv \mathrm{d} / \mathrm{d} \ln (a)$. We will demonstrate that the late time evolutionary scenario crucially depends on the value of the non-minimal coupling constant.

\section{FRW MODEL WITH THE NON-MINIMALLY COUPLED PHANTOM SCALAR FIELD}

We assume the flat model with the FRW geometry, i.e., the line element has the form

$$
\mathrm{d} s^{2}=-\mathrm{d} t^{2}+a^{2}(t)\left[\mathrm{d} r^{2}+r^{2}\left(\mathrm{~d} \theta^{2}+\sin ^{2} \theta \mathrm{d} \varphi^{2}\right)\right],
$$

where $0 \leq \varphi \leq 2 \pi, 0 \leq \theta \leq \pi$ and $0 \leq r \leq \infty$ are comoving coordinates, $t$ stands for the cosmological time. It is also assumed that a source of gravity is the phantom scalar field $\psi$ with an arbitrary coupling constant $\xi$. The dynamics is governed by the action

$$
S=\frac{1}{2} \int \mathrm{d}^{4} x \sqrt{-g}\left(m_{p}^{2} R+\left(g^{\mu \nu} \psi_{\mu} \psi_{\nu}+\xi R \psi^{2}-2 U(\psi)\right)\right)
$$

where $m_{p}^{2}=(8 \pi G)^{-1}$; for simplicity and without lost of generality we assume $4 \pi G / 3=1$ and $U(\psi)$ is a scalar field potential. After dropping the full derivatives with respect to time, rescaling the time variable to the conformal time $\mathrm{d} \eta=\mathrm{d} t / a$ we obtain the energy conservation condition

$$
\mathcal{E}=\frac{1}{2} \dot{a}^{2}+\frac{1}{2} a^{2} \dot{\psi}^{2}+3 \xi \dot{a}^{2} \psi^{2}+6 \xi \dot{a} \dot{\psi} a \psi-a^{4} U(\psi)-\rho_{m, 0} a=\rho_{r, 0}
$$

where $\rho_{r, 0}$ and $\rho_{m, 0}$ are the constants corresponding to the radiation and the matter in the model, respectively, and dot denotes differentiation with respect to conformal time. We can also express this condition as a

$$
\frac{1}{2} H^{2}=\rho_{\psi}+\rho_{r}+\rho_{m}
$$


where $H$ is the Hubble function, $\rho_{\psi}$ is the energy density of a phantom scalar field, $\rho_{r} \propto a^{-4}$ is the energy density of radiation and $\rho_{m} \propto a^{-3}$ is the density of matter.

The Euler-Lagrange equation reduces to

$$
\left\{\begin{array}{l}
a^{2} \ddot{\psi}+6 \xi \ddot{a} a \psi=-2 \dot{a} \dot{\psi} a+a^{4} U(\psi), \\
\ddot{a}\left(1+6 \xi \psi^{2}\right)+6 \xi \ddot{\psi} a \psi=a \dot{\psi}^{2}(1-6 \xi)-12 \xi \dot{a} \dot{\psi} \psi+4 a^{3} U(\psi)+\rho_{m, 0} .
\end{array}\right.
$$

It is easy to check that for the conformal coupling $\xi=1 / 6$, quadratic potential function, the rescaled scalar field variable $\psi=\phi / a$ and $\rho_{m, 0}=0$ this system reduces to well known one.

It would be useful to change

$$
\begin{aligned}
\dot{\psi} & =\frac{\dot{a}}{a} \psi^{\prime}, \\
\ddot{\psi} & =\frac{\ddot{a}}{a} \psi^{\prime}+\frac{\dot{a}^{2}}{a^{2}}\left(\psi^{\prime \prime}-\psi^{\prime}\right),
\end{aligned}
$$

where a dot denotes differentiation with respect to the conformal time and a prime with respect to a natural logarithm of the scale factor. Equations of motion (5) are in the form

$$
\left\{\begin{array}{l}
\frac{\ddot{a}}{a^{3}}\left(\psi^{\prime}+6 \xi \psi\right)+\frac{\dot{a}^{2}}{a^{4}}\left(\psi^{\prime \prime}+\psi^{\prime}\right)=U^{\prime}(\psi), \\
\frac{\ddot{a}}{a^{3}}\left(1+6 \xi \psi\left(\psi^{\prime}+\psi\right)\right)+6 \xi \psi \frac{\dot{a}^{2}}{a^{4}}\left(\psi^{\prime \prime}+\psi^{\prime}\right)=\frac{\dot{a}^{2}}{a^{4}} \psi^{\prime 2}(1-6 \xi)+4 U(\psi)+\rho_{m},
\end{array}\right.
$$

where

$$
\frac{\dot{a}^{2}}{a^{4}}=2 \frac{U(\psi)+\rho_{r}+\rho_{m}}{1+(1-6 \xi) \psi^{\prime 2}+6 \xi\left(\psi^{\prime}+\psi\right)^{2}} .
$$

After eliminating the scale factor and it derivatives we obtain

$$
\begin{aligned}
& \left(\psi^{\prime \prime}+\psi^{\prime}\right)\left[1+6 \xi(1-6 \xi) \psi^{2}\right]+\psi^{\prime 2}(1-6 \xi)\left(\psi^{\prime}+6 \xi \psi\right)+ \\
& +\frac{1}{2} \frac{1+(1-6 \xi) \psi^{\prime 2}+6 \xi\left(\psi^{\prime}+\psi\right)^{2}}{U(\psi)+\rho_{r}+\rho_{m}}\left[\left(4 U(\psi)+\rho_{m}\right)\left(\psi^{\prime}+6 \xi \psi\right)-U^{\prime}(\psi)\left(1+6 \xi \psi\left(\psi^{\prime}+\psi\right)\right)\right]=0
\end{aligned}
$$

where a prime denotes the differentiation with respect to a natural logarithm of the scale factor.

The dynamical systems methods offer a possibility of analyzing all evolutional paths for all admissible initial conditions in a phase space. They are represented by the trajectories in the phase space. The structure of the phase space is organized by the trajectories and limit sets. The critical points of the system are such points in the phase space for which right-hand sides of the dynamical system vanishes at these points. If the system is non-linear we can linearize it at these critical points and the famous Hartman-Grobman theorem guarantees that linearized system is a good approximation of the original dynamical system in the vicinity of this point. The type of a critical point is also characterized by a linearization matrix. It can be determined from the characteristic equation det $|A-\lambda \mathbf{1}|=0$, where $A$ is the linearization matrix. The critical point is the global attractor if all real parts of the eigenvalues are negative, i.e., $\operatorname{Re} \lambda_{i}<0 \forall_{i}$.

Introducing the new variable $y=\psi^{\prime}$ we can represent equation (8) as the autonomous dynamical system

$$
\begin{aligned}
\psi^{\prime} & =y \\
y^{\prime} & =-y-y^{2}(y+6 \xi \psi) \frac{1-6 \xi}{1+6 \xi \psi^{2}(1-6 \xi)}- \\
& -\frac{1}{2} \frac{1+(1-6 \xi) y^{2}+6 \xi(y+\psi)^{2}}{1+6 \xi \psi^{2}(1-6 \xi)}\left[\frac{\left(4 U(\psi)+\rho_{m}\right)(y+6 \xi \psi)-U^{\prime}(\psi)(1+6 \xi \psi(y+\psi))}{U(\psi)+\rho_{r}+\rho_{m}}\right] \\
\rho_{r}^{\prime} & =-4 \rho_{r} \\
\rho_{m}^{\prime} & =-3 \rho_{m},
\end{aligned}
$$

where we have $\rho_{m}=\rho_{m, 0} a^{-3}$ and $\rho_{r}=\rho_{r, 0} a^{-4}$.

The equation of state can be directly determined from the dynamics

$$
\begin{aligned}
w_{\text {eff }}=2\{ & -\xi\left(\psi^{\prime}+\psi\right)^{2}+\frac{1}{H^{2}}\left(2 \xi \psi U^{\prime}(\psi)-U(\psi)+\frac{1}{3} \rho_{r}\right)+ \\
& \left.+2 \frac{1-6 \xi}{1+6 \xi \psi^{2}(1-6 \xi)}\left[-\frac{1}{4} \psi^{\prime 2}\left(1+2 \xi \psi^{2}(1-6 \xi)\right)+\xi \frac{\psi^{2}}{H^{2}}\left(\rho_{m}+4 U(\psi)-6 \xi \psi U^{\prime}(\psi)\right)\right]\right\} .
\end{aligned}
$$


where

$$
H^{2}=2 \frac{U(\psi)+\rho_{r}+\rho_{m}}{1+(1-6 \xi) \psi^{\prime 2}+6 \xi\left(\psi^{\prime}+\psi\right)^{2}} .
$$

is the counterpart of the Friedmann first integral.

On the invariant submanifold we change the "time" variable

$$
\varepsilon \frac{\mathrm{d}}{\mathrm{d} \sigma}=U(\psi)\left(1+6 \xi \psi^{2}(1-6 \xi)\right) \frac{\mathrm{d}}{\mathrm{d} \ln (a)}
$$

where $\varepsilon=1$ when $U(\psi)\left(1+6 \xi \psi^{2}(1-6 \xi)\right)>0$ and $\varepsilon=-1$ when $U(\psi)\left(1+6 \xi \psi^{2}(1-6 \xi)\right)<0$. The dynamical system is in the form

$$
\begin{aligned}
\varepsilon \dot{\psi}= & y U(\psi)\left(1+6 \xi \psi^{2}(1-6 \xi)\right), \\
\varepsilon \dot{y}= & -y U(\psi)\left(1+6 \xi \psi^{2}(1-6 \xi)\right)-(1-6 \xi) y^{2}(y+6 \xi \psi) U(\psi)- \\
& -\frac{1}{2}\left(1+(1-6 \xi) y^{2}+6 \xi(y+\psi)^{2}\right)\left(4 U(\psi)(y+6 \xi \psi)-U^{\prime}(\psi)(1+6 \xi \psi(y+\psi))\right) .
\end{aligned}
$$

In the generic case critical points of a dynamical system are located:

1) $y_{0}=0$ and $\left(1+6 \xi \psi_{0}^{2}\right)\left(24 \xi \psi_{0} U\left(\psi_{0}\right)-U^{\prime}\left(\psi_{0}\right)\left(1+6 \xi \psi_{0}^{2}\right)\right)=0$;

2) $\psi_{0}^{2}=\frac{1}{6 \xi(6 \xi-1)}$ for $\xi>1 / 6$ or $\xi<0$ and $y_{0}$ is a solution of the cubic equation

$$
A(\xi) y^{3}+B(\xi) y^{2}+C(\xi) y+D(\xi)=0
$$

where for a given form of the potential function, $A(\xi), B(\xi), C(\xi), D(\xi)$ are functions of the coupling constant $\xi$ only.

For our further investigations the critical point (1) is especially interesting. At this point the trace and the determinant of the linearization matrix are

$$
\begin{gathered}
\operatorname{tr} A=-U\left(\psi_{0}\right)\left(1+6 \xi \psi_{0}^{2}(1-6 \xi)\right)-\frac{1}{2}\left(1+6 \xi \psi_{0}^{2}\right)\left(4 U\left(\psi_{0}\right)-6 \xi \psi_{0} U^{\prime}\left(\psi_{0}\right)\right) \\
\operatorname{det} A=-\frac{1}{2}\left(1+6 \xi \psi_{0}^{2}\right)\left(12 \xi \psi_{0} U^{\prime}\left(\psi_{0}\right)+24 \xi U\left(\psi_{0}\right)-U^{\prime \prime}\left(\psi_{0}\right)\left(1+6 \xi \psi_{0}^{2}\right)\right) U\left(\psi_{0}\right)\left(1+6 \xi \psi_{0}^{2}(1-6 \xi)\right)
\end{gathered}
$$

The type of behavior around this point crucially depends on the sign of $\Delta=(\operatorname{tr} A)^{2}-4 \operatorname{det} A$. We are interested in the case $\xi>0$. Note that at this critical point

$$
L=\frac{U^{\prime}\left(\psi_{0}\right)}{U\left(\psi_{0}\right)}=\frac{24 \xi \psi_{0}}{1+6 \xi \psi_{0}^{2}}
$$

we obtain that

$$
\Delta=\left(U\left(\psi_{0}\right)\right)^{2}\left(\left(1+6 \xi \psi_{0}^{2}(1-6 \xi)\right)\left(9\left(1+6 \xi \psi_{0}^{2}(1-6 \xi)\right)-48\left(18 \xi^{2} \psi_{0}^{2}+\xi-\Gamma 24 \xi^{2} \psi_{0}^{2}\right)\right)\right)
$$

The general condition for $\Delta \leq 0$ is

$$
\Gamma=\frac{U\left(\psi_{0}\right) U^{\prime \prime}\left(\psi_{0}\right)}{U^{\prime}\left(\psi_{0}\right)^{2}} \leq \frac{1}{24 \xi^{2} \psi_{0}^{2}}\left(18 \xi^{2} \psi_{0}^{2}+\xi-\frac{9}{48}\left(1+6 \xi \psi_{0}^{2}(1-6 \xi)\right)\right) .
$$

\section{COSMOLOGY WITH NON-MINIMAL COUPLING AS A DYNAMICAL SYSTEM}

In this section we concentrate on investigation of the dynamics of the models with the quadratic potential because for other most popular forms of the potential functions there are attractors only for the negative coupling parameter. This case seems to be less interesting physically [10]. For the quadratic potential $U(\psi)=\frac{1}{2} m^{2} \psi^{2}, m^{2}=1$, the dynamical system on the invariant submanifold reduces to

$$
\begin{aligned}
& \varepsilon \dot{\psi}=\frac{1}{2} y \psi^{2}\left(1+6 \xi \psi^{2}(1-6 \xi)\right), \\
& \varepsilon \dot{y}=-\frac{1}{2} y \psi^{2}\left(1+(1-6 \xi)\left(6 \xi \psi(\psi+y)+y^{2}\right)\right)-\frac{1}{2} \psi\left(1+(1-6 \xi) y^{2}+6 \xi(y+\psi)^{2}\right)\left(2 y \psi(1-3 \xi)+6 \xi \psi^{2}-1\right),
\end{aligned}
$$


where a dot means differentiation with respect to $\frac{\mathrm{d}}{\mathrm{d} \sigma}=\frac{1}{2} \psi^{2}\left(1+6 \xi \psi^{2}(1-6 \xi)\right) \frac{\mathrm{d}}{\mathrm{d} \ln (a)}$.

The linearization matrix of system (16) at the critical point $y_{0}=0$ and $\psi_{0}^{2}=\frac{1}{6 \xi}$ is

$$
A=\left(\begin{array}{cc}
0 & \frac{1-3 \xi}{6 \xi} \\
-2 & -\frac{1-3 \xi}{2 \xi}
\end{array}\right) \text {. }
$$

The eigenvalues of the linearization matrix are:

$$
\lambda_{1,2}=\frac{1}{4 \xi}\left[-(1-3 \xi) \pm \frac{\sqrt{3}}{3} \sqrt{\Delta}\right]
$$

where $\Delta=(1-3 \xi)(3-25 \xi)$. The type of the critical point depends on the value of $\Delta$.

- for $0<\xi<\frac{3}{25}$ :

$\Delta>0$ and both eigenvalues are real and negative, the critical point is a stable node (Fig 1);

- for $\frac{3}{25}<\xi<\frac{1}{3}$ :

$\Delta<0$ we have two complex eigenvalues and the critical point is of a focus type (Fig 2);

- for $\xi>\frac{1}{3}$ :

$\Delta>0$ and $\lambda_{1}>0, \lambda_{2}<0$ and the critical point is of a saddle type.

- for $\xi=\frac{3}{25}$ or $\xi=\frac{1}{3}$ :

$\Delta=0$ and critical point is degenerated (Fig 3$)$.

The eigenvectors are

$$
v_{1,2}=\left[\begin{array}{c}
1 \\
-\frac{3}{2} \pm \frac{\sqrt{3}}{2} \sqrt{\frac{3-25 \xi}{1-3 \xi}}
\end{array}\right] .
$$

They are helpful in the construction of the exact solution of the linearized system. For now, we restrict ourselves to the case of $0<\xi<3 / 25$, for other cases the construction of the linearized solution is similar.

$$
\begin{aligned}
& \vec{x}(\sigma)=\vec{x}(0) \exp \left\{\sigma\left(\begin{array}{cc}
0 & \frac{1-3 \xi}{6 \xi} \\
-2 & -\frac{1-3 \xi}{2 \xi}
\end{array}\right)\right\}= \\
& \left(\begin{array}{cc}
1 \\
-\frac{3}{2}+\frac{\sqrt{3}}{2} \sqrt{\frac{3-25 \xi}{1-3 \xi}}-\frac{3}{2}-\frac{\sqrt{3}}{2} \sqrt{\frac{3-25 \xi}{1-3 \xi}}
\end{array}\right)\left(\begin{array}{cc}
\exp \lambda_{1} \sigma & 0 \\
0 & \exp \lambda_{2} \sigma
\end{array}\right)\left(\begin{array}{cc}
\frac{1}{2}+\frac{\sqrt{3}}{2} \sqrt{\frac{3-25 \xi}{1-3 \xi}} & \frac{\sqrt{3}}{3} \sqrt{\frac{3-25 \xi}{1-3 \xi}} \\
\frac{1}{2}-\frac{\sqrt{3}}{2} \sqrt{\frac{3-25 \xi}{1-3 \xi}} & -\frac{\sqrt{3}}{3} \sqrt{\frac{3-25 \xi}{1-3 \xi}}
\end{array}\right)\left(\begin{array}{l}
x_{0} \\
y_{0}
\end{array}\right)
\end{aligned}
$$

where $\sigma=\frac{6 \xi}{1-3 \xi} \ln a$ and $x_{0}=\psi_{i}-\psi_{0}, y_{0}=\psi_{i}^{\prime}-\psi_{0}^{\prime}$ are initial conditions. The exact solutions are

$$
\begin{aligned}
\psi-\psi_{0} & =a^{\alpha_{1}}\left[\frac{1}{2} x_{0}+\sqrt{3} \sqrt{\frac{1-3 \xi}{3-25 \xi}}\left(\frac{1}{2} x_{0}+\frac{1}{3} y_{0}\right)\right]+a^{\alpha_{2}}\left[\frac{1}{2} x_{0}-\sqrt{3} \sqrt{\frac{1-3 \xi}{3-25 \xi}}\left(\frac{1}{2} x_{0}+\frac{1}{3} y_{0}\right)\right], \\
\psi^{\prime}-\psi_{0}^{\prime} & =\alpha_{1} a^{\alpha_{1}}\left[\frac{1}{2} x_{0}+\sqrt{3} \sqrt{\frac{1-3 \xi}{3-25 \xi}}\left(\frac{1}{2} x_{0}+\frac{1}{3} y_{0}\right)\right]+\alpha_{2} a^{\alpha_{2}}\left[\frac{1}{2} x_{0}-\sqrt{3} \sqrt{\frac{1-3 \xi}{3-25 \xi}}\left(\frac{1}{2} x_{0}+\frac{1}{3} y_{0}\right)\right],
\end{aligned}
$$

where $x_{0}, y_{0}$ are initial conditions, $\alpha_{1}=-\frac{1}{2}\left(3-\sqrt{3} \sqrt{\frac{3-25 \xi}{1-3 \xi}}\right)$ and $\alpha_{2}=-\frac{1}{2}\left(3+\sqrt{3} \sqrt{\frac{3-25 \xi}{1-3 \xi}}\right)$. Notice that these solutions are also valid for $\xi>1 / 3$ (a saddle type critical point).

For the degenerated case $\xi=3 / 25$, equations (20) and (21) simplify to

$$
\begin{aligned}
\psi-\psi_{0} & =x_{0} a^{-3 / 2}, \\
\psi^{\prime}-\psi_{0}^{\prime} & =-\frac{3}{2} x_{0} a^{-3 / 2} .
\end{aligned}
$$


The same procedure can be applied to construction the linearized solution for $\Delta<0$. Instead we can use these solutions if we notice that for $3 / 25<\xi<1 / 3$

$$
\begin{aligned}
& \alpha_{1}=-\frac{3}{2}+i \frac{\sqrt{3}}{2} \sqrt{\frac{25 \xi-3}{1-3 \xi}}=-\frac{3}{2}+i \alpha, \\
& \alpha_{2}=-\frac{3}{2}-i \frac{\sqrt{3}}{2} \sqrt{\frac{25 \xi-3}{1-3 \xi}}=-\frac{3}{2}-i \alpha,
\end{aligned}
$$

then we have

$$
\begin{aligned}
\psi-\psi_{0} & =a^{-3 / 2}\left[x_{0} \cos (\alpha \ln a)+3 \alpha^{-1} \sin (\alpha \ln a)\left(\frac{1}{2} x_{0}+\frac{1}{3} y_{0}\right)\right], \\
\psi^{\prime}-\psi_{0}^{\prime} & =a^{-3 / 2}\left[y_{0} \cos (\alpha \ln a)-\alpha x_{0} \sin (\alpha \ln a)-\frac{9}{2} \alpha^{-1} \sin (\alpha \ln a)\left(\frac{1}{2} x_{0}+\frac{1}{3} y_{0}\right)\right],
\end{aligned}
$$

where $x_{0}, y_{0}$ are initial conditions, and $\alpha$ depends on the coupling constant $\xi$ only.

The substitution of $\psi$ and $\psi^{\prime}$ gives us a general formula for $w_{\text {eff }}$ around the critical point of a given type.

On the invariant submanifold and for quadratic potential function $U(\psi)=\frac{1}{2} \psi^{2}$ formula for equation of state parameter reduces to

$$
w_{X}=\frac{2}{1+6 \xi \psi^{2}(1-6 \xi)}\left\{\frac{1}{2}\left(1+2 \xi \psi^{2}(1-6 \xi)\right)-(1-2 \xi)\left(1+(1-6 \xi) \psi^{2}\right)-4 \xi(1-3 \xi)\left(\psi^{\prime}+\psi\right)^{2}\right\}
$$

In the case when the critical point is a stable node $(0<\xi<3 / 25)$ we have

$$
w_{X}^{\text {mon }}=\frac{-(1-3 \xi)+f_{1}(\xi, a) a^{-3 / 2}+f_{2}(\xi, a) a^{-3}}{(1-3 \xi)+6 \xi(1-6 \xi) \psi_{0}\left(A a^{\alpha_{l}}+B a^{-\alpha_{l}}\right) a^{-3 / 2}+3 \xi(1-6 \xi)\left(A a^{\alpha_{l}}+B a^{-\alpha_{l}}\right)^{2} a^{-3}},
$$

where $\psi_{0}^{2}=\frac{1}{6 \xi}, \alpha_{l}=\frac{\sqrt{3}}{2} \sqrt{\frac{3-25 \xi}{1-3 \xi}}$

$$
\begin{aligned}
& A=\frac{1}{2} x_{0}+\sqrt{3} \sqrt{\frac{1-3 \xi}{3-25 \xi}}\left(\frac{1}{2} x_{0}+\frac{1}{3} y_{0}\right), \\
& B=\frac{1}{2} x_{0}-\sqrt{3} \sqrt{\frac{1-3 \xi}{3-25 \xi}}\left(\frac{1}{2} x_{0}+\frac{1}{3} y_{0}\right),
\end{aligned}
$$

$x_{0}$ and $y_{0}$ are the initial conditions for $\psi$ and $\psi^{\prime}$, respectively, and functions $f_{1}$ and $f_{2}$ are given by

$$
\begin{aligned}
f_{1}(\xi, a) & =2 \xi \psi_{0}\left(\left(3(1-4 \xi)-4 \alpha_{l}(1-3 \xi)\right) A a^{\alpha_{l}}+\left(3(1-4 \xi)+4 \alpha_{l}(1-3 \xi)\right) B a^{-\alpha_{l}}\right) \\
f_{2}(\xi, a) & =\left(-\frac{3}{4}(3-4 \xi)+15 \xi(1-2 \xi)\right)\left(A a^{\alpha_{l}}+B a^{-\alpha_{l}}\right)^{2}+ \\
& +\alpha_{l}(3(1-4 \xi)-8 \xi(1-3 \xi))\left(A^{2} a^{2 \alpha_{l}}-B^{2} a^{-2 \alpha_{l}}\right)-\alpha_{l}^{2}(1-4 \xi)\left(A a^{\alpha_{l}}-B a^{-\alpha_{l}}\right)^{2}
\end{aligned}
$$

This case can be slightly simplified if we notice that there are two independent directions leading to the critical point of the stable node type

1. $B=\frac{1}{2} x_{0}-\sqrt{3} \sqrt{\frac{1-3 \xi}{3-25 \xi}}\left(\frac{1}{2} x_{0}+\frac{1}{3} y_{0}\right)=0$,

2. $A=\frac{1}{2} x_{0}+\sqrt{3} \sqrt{\frac{1-3 \xi}{3-25 \xi}}\left(\frac{1}{2} x_{0}+\frac{1}{3} y_{0}\right)=0$.

Then

$$
w_{\text {mon }}=-\frac{(1-3 \xi)+\sqrt{6 \xi}(1-2 \xi) x_{0} a^{\alpha_{i}}+\left(3 \xi(1-2 \xi)+8 \xi(1-3 \xi) \alpha_{i}+(1-4 \xi) \alpha_{i}^{2}\right) x_{0}^{2} a^{2 \alpha_{i}}}{(1-3 \xi)+\sqrt{6 \xi}(1-6 \xi) x_{0} a^{\alpha_{i}}+3 \xi(1-6 \xi) x_{0}^{2} a^{2 \alpha_{i}}}
$$


Using general formula

$$
\rho_{X}=\rho_{X, 0} a^{-3} \exp \left\{-3 \int_{1}^{a} \frac{w\left(a^{\prime}\right)}{a^{\prime}} \mathrm{d} a^{\prime}\right\}
$$

we can calculate dark energy density in the case of monotonic approach to deSitter state

$$
\rho_{X}=\rho_{X, 0} A\left(B_{0}+B_{1} a^{\alpha_{i}}+B_{2} a^{2 \alpha_{i}}\right)^{\beta} \exp \left\{-\gamma \arctan \left(\sqrt{1-6 \xi}\left(1+\sqrt{6 \xi} x_{0} a^{\alpha_{i}}\right)\right)\right\}
$$

where

$$
A=\left(B_{0}+B_{1}+B_{2}\right)^{-\beta} \exp \left\{\gamma \arctan \left(\sqrt{1-6 \xi}\left(1+\sqrt{6 \xi} x_{0}\right)\right)\right\}
$$

$B_{0}=1-3 \xi, B_{1}=\sqrt{6 \xi}(1-6 \xi) x_{0}, B_{2}=3 \xi(1-6 \xi) x_{0}^{2}$ and

$$
\begin{gathered}
\beta=\frac{12 \xi^{2}+8 \xi(1-3 \xi) \alpha_{i}+(1-4 \xi) \alpha_{i}^{2}}{2 \xi(1-6 \xi) \alpha_{i}}, \\
\gamma=\frac{-12 \xi^{2}+8 \xi(1-3 \xi) \alpha_{i}+(1-4 \xi) \alpha_{i}^{2}}{\xi \sqrt{1-6 \xi} \alpha_{i}},
\end{gathered}
$$

$\alpha_{1}=-\frac{1}{2}\left(3-\sqrt{3} \sqrt{\frac{3-25 \xi}{1-3 \xi}}\right)$ and $\alpha_{2}=-\frac{1}{2}\left(3+\sqrt{3} \sqrt{\frac{3-25 \xi}{1-3 \xi}}\right)$

In the case of damped oscillatory approach to the critical point $(3 / 25<\xi<1 / 3)$ and for critical point $\psi_{0}^{2}=1 / 6 \xi$, $\psi_{0}^{\prime}=0$ we have general equation of state parameter in the form

$$
w_{X}^{\mathrm{osc}}=\frac{-(1-3 \xi)+g_{1}(\xi, a) a^{-3 / 2}+g_{2}(\xi, a) a^{-3}}{(1-3 \xi)+6 \xi(1-6 \xi) \psi_{0} h(\xi, a) a^{-3 / 2}+3 \xi(1-6 \xi) h^{2}(\xi, a) a^{-3}}
$$

where the functions $h, g_{1}$ and $g_{2}$ are

$$
\begin{aligned}
h(\xi, a)= & x_{0} \cos \left(\alpha_{\mathrm{osc}} \ln a\right)+\frac{3}{\alpha_{\mathrm{osc}}} \sin \left(\alpha_{\mathrm{osc}} \ln a\right)\left(\frac{1}{2} x_{0}+\frac{1}{3} y_{0}\right) \\
g_{1}(\xi, a)= & 2 \xi \psi_{0}\left((1-6 \xi) h(\xi, a)-4(1-3 \xi)\left(\left(x_{0}+y_{0}\right) \cos \left(\alpha_{\mathrm{osc}} \ln a\right)-\alpha_{\mathrm{osc}} x_{0} \sin \left(\alpha_{\mathrm{osc}} \ln a\right)-\right.\right. \\
& \left.\left.\quad-\frac{3}{2 \alpha_{\mathrm{osc}}} \sin \left(\alpha_{\mathrm{osc}} \ln a\right)\left(\frac{1}{2} x_{0}+\frac{1}{3} y_{0}\right)\right)\right) \\
g_{2}(\xi, a)= & \xi(1-6 \xi) h^{2}(\xi, a)- \\
- & (1-2 \xi)(1-6 \xi)\left(y_{0} \cos \left(\alpha_{\mathrm{osc}} \ln a\right)-\alpha_{\mathrm{osc}} x_{0} \sin \left(\alpha_{\mathrm{osc}} \ln a\right)-\frac{9}{2 \alpha_{\mathrm{osc}}} \sin \left(\alpha_{\mathrm{osc}} \ln a\right)\left(\frac{1}{2} x_{0}+\frac{1}{3} y_{0}\right)\right)^{2}- \\
- & 4 \xi(1-3 \xi)\left(\left(x_{0}+y_{0}\right) \cos \left(\alpha_{\mathrm{osc}} \ln a\right)-\alpha_{\mathrm{osc}} x_{0} \sin \left(\alpha_{\mathrm{osc}} \ln a\right)-\frac{3}{2 \alpha_{\mathrm{osc}}} \sin \left(\alpha_{\mathrm{osc}} \ln a\right)\left(\frac{1}{2} x_{0}+\frac{1}{3} y_{0}\right)\right)^{2},
\end{aligned}
$$

where $\alpha_{\mathrm{osc}}=\frac{\sqrt{3}}{2} \sqrt{\frac{25 \xi-3}{1-3 \xi}}$ and $x_{0}, y_{0}$ and $\psi_{0}$ have their usual meaning.

In the case of conformal coupling and assuming that $y_{0}=-\frac{3}{2} x_{0}$, equation of state parameter (35) simplifies to

$$
w_{X ; \xi=\frac{1}{6}}^{\mathrm{osc}}=-1+\frac{2}{3} a^{-3 / 2}\left(\cos \left(\frac{\sqrt{7}}{2} \ln a\right)+\sqrt{7} \sin \left(\frac{\sqrt{7}}{2} \ln a\right)\right) x_{0}-\frac{1}{6} a^{-3}(4-3 \cos (\sqrt{7} \ln a)+\sqrt{7} \sin (\sqrt{7} \ln a)) x_{0}^{2} .
$$

This expression can be easy integrated and we receive dark energy density in the case of conformally coupled phantom scalar field

$$
\begin{aligned}
\rho_{X ; \xi=\frac{1}{6}}= & \rho_{X, 0} \exp \left(-\frac{1}{48}\left(120-29 x_{0}\right) x_{0}\right) \exp \left(\frac{1}{2} a^{-3 / 2}\left(5 \cos \left(\frac{\sqrt{7}}{2} \ln a\right)+\sqrt{7} \sin \left(\frac{\sqrt{7}}{2} \ln a\right)\right) x_{0}\right) \\
& \exp \left(\frac{1}{48} a^{-3}(-32+3 \cos (\sqrt{7} \ln a)-9 \sqrt{7} \sin (\sqrt{7} \ln a)) x_{0}^{2}\right)
\end{aligned}
$$

where $x_{0}$ and $\rho_{X, 0}$ are two constants fitted from observational data. 
It is interesting to consider the special case of minimal coupling $\xi=0$. The dynamical system is in the form

$$
\left\{\begin{array}{l}
\frac{\mathrm{d} \psi}{\mathrm{d} \tau}=2 y \frac{U(\psi)}{U^{\prime}(\psi)} \\
\frac{\mathrm{d} y}{\mathrm{~d} \tau}=-\left(1+y^{2}\right)\left(6 y \frac{U(\psi)}{U^{\prime}(\psi)}-1\right)
\end{array}\right.
$$

where $\frac{\mathrm{d}}{\mathrm{d} \tau}=\frac{2}{U^{\prime}(\psi)} \frac{\mathrm{d}}{\mathrm{d} \sigma}=2 \frac{U(\psi)}{U^{\prime}(\psi)} \frac{\mathrm{d}}{\mathrm{d} \ln a}$. Above dynamical system can be easily integrated

$$
\begin{gathered}
\mathrm{d} y \frac{1}{1+y^{2}}=-3 \mathrm{~d} \psi+\mathrm{d} \tau, \\
y=\psi^{\prime}=\tan \left(-3 \psi+\Delta \tau+C_{0}\right),
\end{gathered}
$$

where

$$
\Delta \tau=\frac{1}{2} \int_{i}^{a} \frac{\mathrm{d} \ln U(\psi)}{\mathrm{d} \psi} \mathrm{d} \ln a^{\prime},
$$

is the "period of time" between initial and final state and $C_{0}$ is the constant of integration. The equation of state parameter assumes very simple form for the minimal coupling

$$
w_{X, \xi=0}=-1-2 \psi^{\prime 2} \text {. }
$$

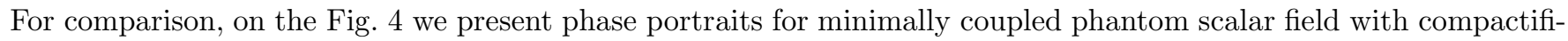
cation at infinity for various potential functions. It is interesting that for every discussed form of the potential there are always trajectories with $w_{X, \xi=0}=-1$ at a certain moment of the evolution.

In current astronomical analysis of the nature of dark energy, the most popular are linear (in the scale factor or the redshift) parametrizations of equation of state parameter. Let us compare equations (27) and (35) with current estimations of coefficients of this parametrizations. Expanding (27) and (35) in to the Taylor series with respect to the scale factor or the redshift at the present epoch we receive polynomial approximation of exact form of the equation of state parameter. As an example we use two linear parametrizations and values of $w_{0}$ and $w_{1}$ estimated by Dicus and Repko [34]. In the Taylor series we have three free parameters $x_{0}, y_{0}$ and $\xi$, but form observations we have only two numbers $w_{0}$ and $w_{1}$, so one free parameter should be removed. Most convenient seems to be assumption that $y_{0}=-\frac{3}{2} x_{0}$. Taking in to account only two first terms in the Taylor series we can solve system on the $\xi$ and $x_{0}$. On the Fig. [5 we present dependence of $x_{0}$ on the coupling parameter $\xi$ for the values of $w_{0}$ and $w_{1}$ form [34]. Intersections of this curves are solutions on $x_{0}$ and $\xi$. It is interesting that the obtained values of the coupling parameter $\xi$ with the assumption $y_{0}=-\frac{3}{2} x_{0}$ are close to the conformal coupling $\xi=\frac{1}{6}$.

\section{ANALYSIS AT INFINITY}

The full description of a dynamical system includes also behavior of its trajectories at infinity. It is performed in tools of the Poincare sphere construction. In this approach we project trajectories from the center of the unit sphere $\mathbf{S}^{2}$ onto the plane $(x, y)$ tangent to $\mathbf{S}^{2}$ either at the north or south pole (for details see [35, p. 265]). Due to this construction infinitely distant points of the plane are mapped into the sphere equator, phase trajectories are mapped into corresponding curves on the sphere but the character of critical points is conserved and new critical points can appear at the equator. Hence the orthogonal projection of any hemisphere onto a tangent plane gives compactified phase portrait on the plane. In practice, polar or projective coordinates are introduced on the plane. Due to this central projection which was introduced by Poincare we can observe how critical points representing asymptotic states of the system are spread out along the equator. Moreover if r.h.s. of the system are given in polynomial form then we have simple test of structural stability of the system. In particular, a vector field on the Poincare sphere will be structurally unstable if there are non-hyperbolic critical points at infinity or if there is a trajectory connecting a saddles on the equator of Poincare sphere. In opposite case if number of critical points and limit cycles is finite dynamical system is structurally stable and therefore generic in the Peixoto sense.

Introducing new variables $(r, \theta)$ with compactification at infinity:

$$
\begin{aligned}
\psi & =\frac{r}{1-r} \cos \theta, \\
y & =\frac{r}{1-r} \sin \theta,
\end{aligned}
$$




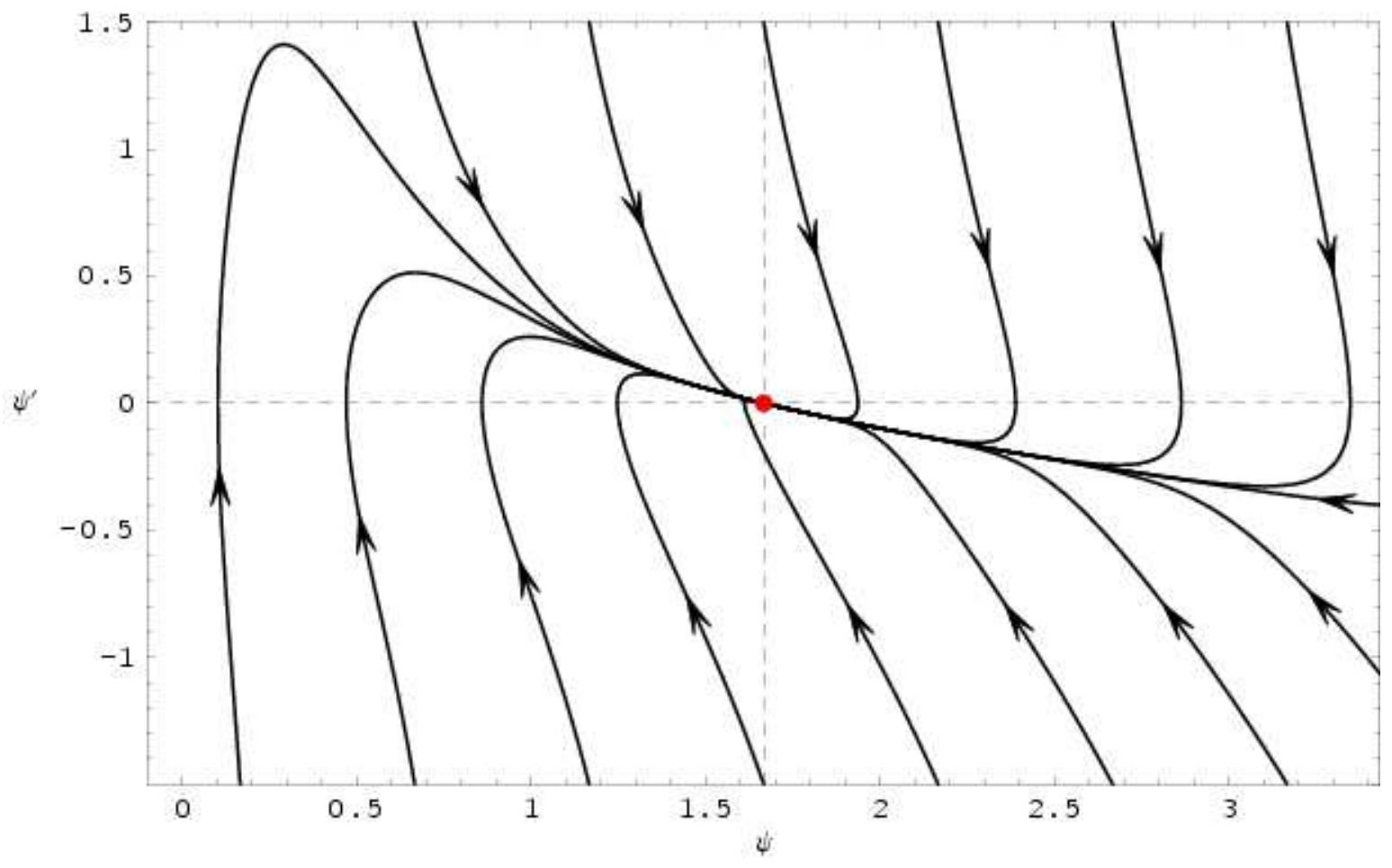

FIG. 1: The phase portrait for $\xi=3 / 50<3 / 25$, it represents general behavior around the critical point of a stable node type, $w_{\text {eff }}=-1$ at this point.

we receive dynamical system in the form

$$
\begin{aligned}
\theta^{\prime}=-\frac{1}{2} & \cos ^{2} \theta\left\{r^{2} \sin ^{2} \theta\left((1-r)^{2}+6 \xi(1-6 \xi) r^{2} \cos ^{2} \theta\right)+\right. \\
& +r^{2} \sin \theta \cos \theta\left((1-r)^{2}+(1-6 \xi)\left(6 \xi r^{2} \cos \theta(\cos \theta+\sin \theta)+r^{2} \sin ^{2} \theta\right)\right)+ \\
& \left.+\left((1-r)^{2}+(1-6 \xi) r^{2} \sin ^{2} \theta+6 \xi r^{2}(\sin \theta+\cos \theta)^{2}\right)\left(2 r^{2} \sin \theta \cos \theta-(1-r)^{2}+6 \xi r^{2} \cos \theta(\cos \theta-\sin \theta)\right)\right\} \\
r^{\prime}=\frac{1}{2} r(1 & -r) \sin \theta \cos \theta\left\{r^{2} \cos ^{2} \theta\left((1-r)^{2}+6 \xi(1-6 \xi) r^{2} \cos ^{2} \theta\right)-\right. \\
& -r^{2} \sin \theta \cos \theta\left((1-r)^{2}+(1-6 \xi)\left(6 \xi r^{2} \cos \theta(\cos \theta+\sin \theta)+r^{2} \sin ^{2} \theta\right)\right)- \\
& \left.-\left((1-r)^{2}+(1-6 \xi) r^{2} \sin ^{2} \theta+6 \xi r^{2}(\sin \theta+\cos \theta)^{2}\right)\left(2 r^{2} \sin \theta \cos \theta-(1-r)^{2}+6 \xi r^{2} \cos \theta(\cos \theta-\sin \theta)\right)\right\}
\end{aligned}
$$

where a prime denotes now differentiation with respect to $\mathrm{d} / \mathrm{d} \tau=(1-r)^{4} \mathrm{~d} / \mathrm{d} \sigma$ (Figs. 6, 7, 8).

The number and character of critical points at infinity depends on the value of $\xi$. In the general case we have critical points $\left(r_{0}=1, \theta_{0}\right): \theta= \pm \pi / 2$ and solution to the equation

$$
6 \xi(7-24 \xi) \sin ^{2} \theta \cos \theta+18 \xi \sin \theta \cos ^{2} \theta+3(1-4 \xi) \sin ^{3} \theta+36 \xi^{2} \cos ^{4} \theta=0 .
$$

This equation can be easily solved for arbitrary coupling constant $\xi$. On Fig. 9 we present location and number of critical point at infinity as a function of coupling constant $\xi$.

\section{CONCLUSIONS}

In this paper we considered the non-minimally coupled phantom scalar field as the FRW model with dark energy driving the acceleration of the current universe. We called it the extended quintessence and formulated the FRW dynamics with this form of dark energy in terms of the autonomous dynamical system. Hence we study all evolutional 


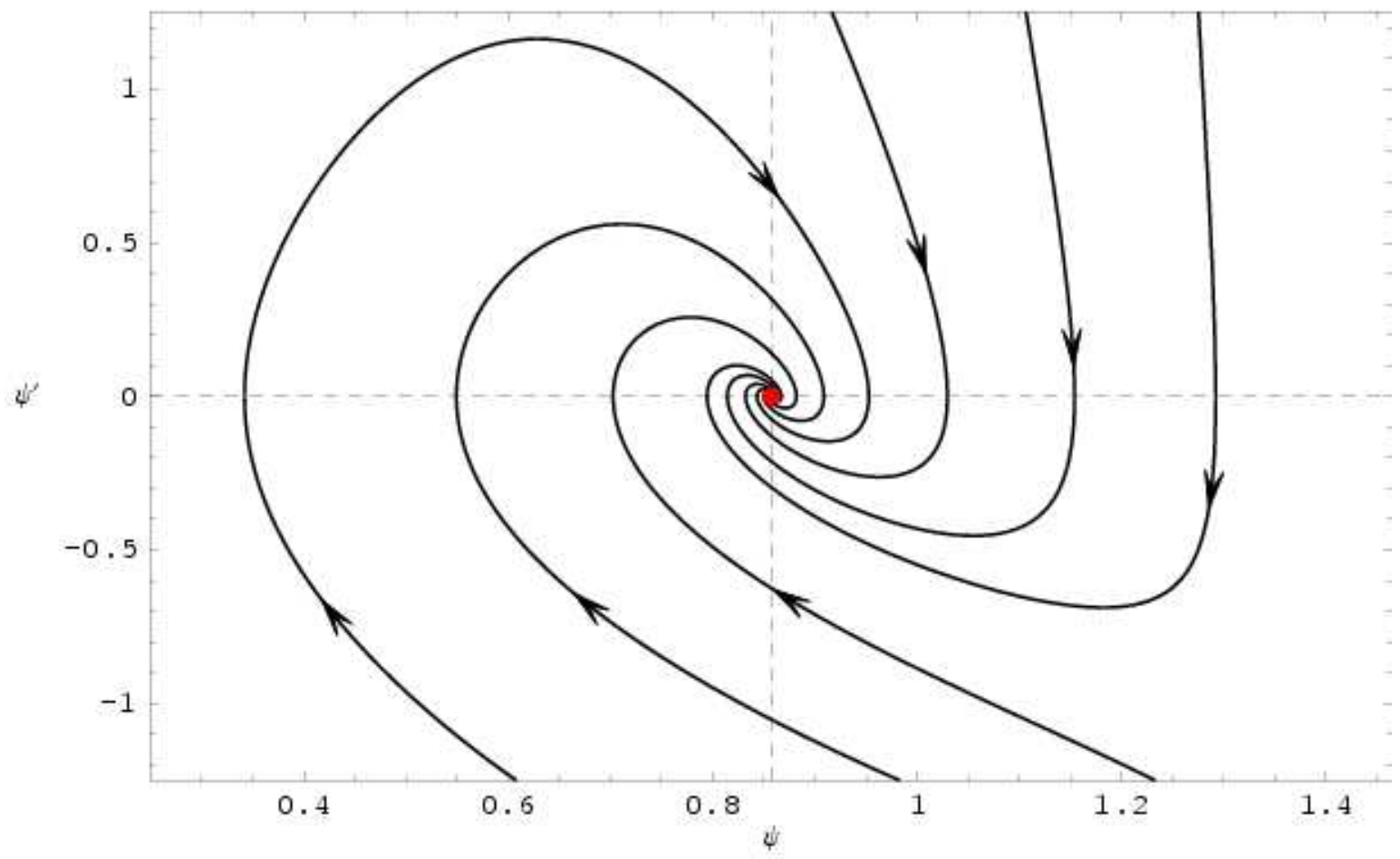

FIG. 2: Typical trajectories on the invariant 2-dimensional submanifold for the quadratic potential function and $\xi=17 / 75$. The phase portrait represents the generic behavior around a focus type critical point. For $3 / 25<\xi \leq 1 / 6$ we have the global attractor of this type in the phase space, for $\xi>1 / 6$ the critical point of this type is no longer a global attractor.

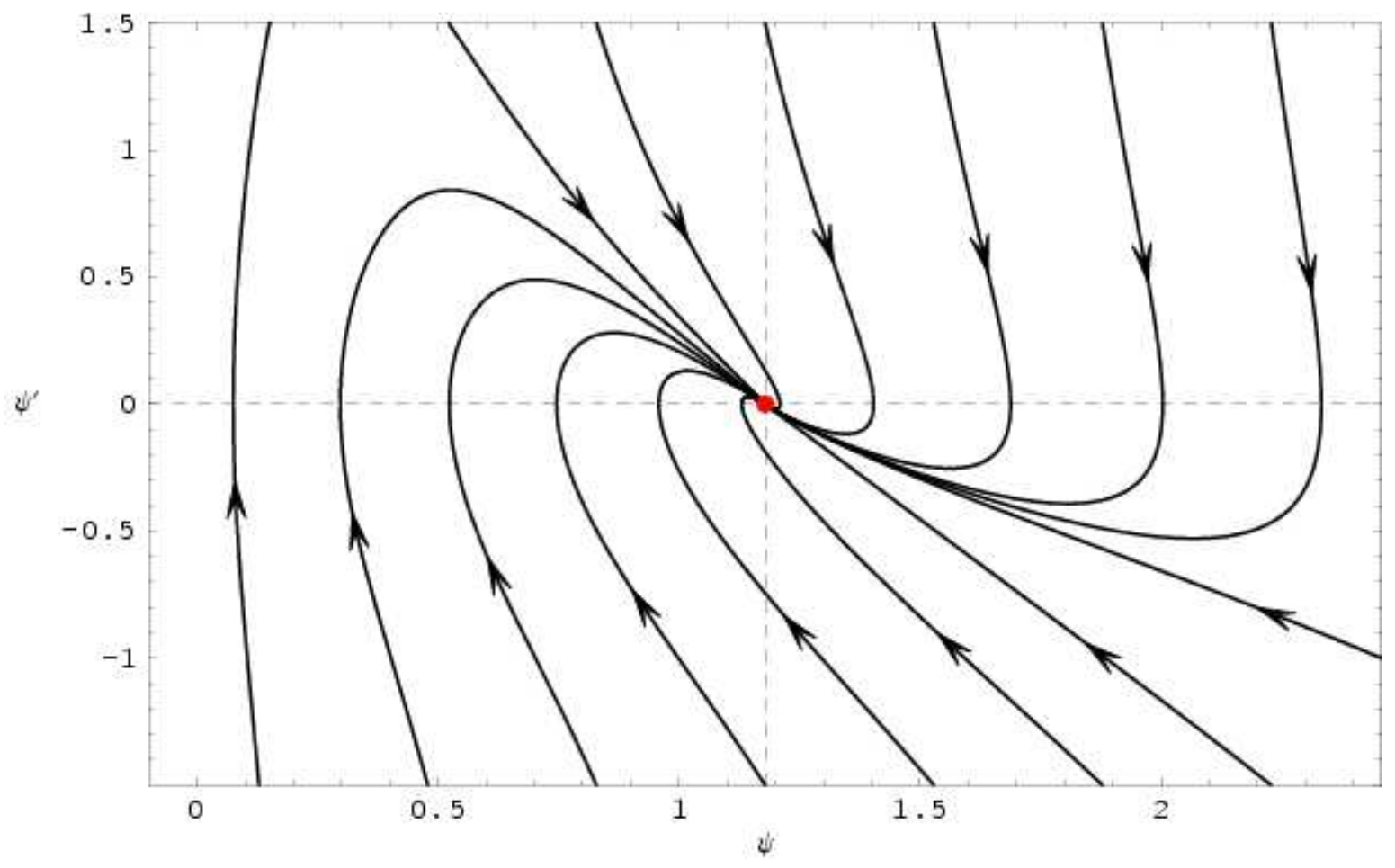

FIG. 3: The phase portrait for the degenerated case $\xi=3 / 25$. 
a)

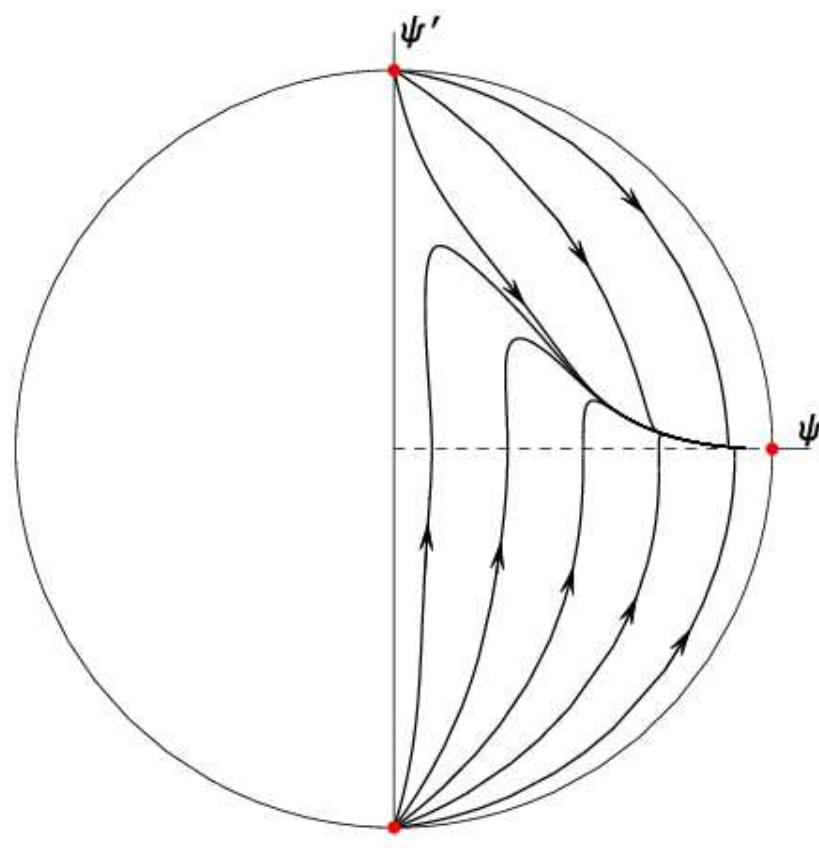

b)



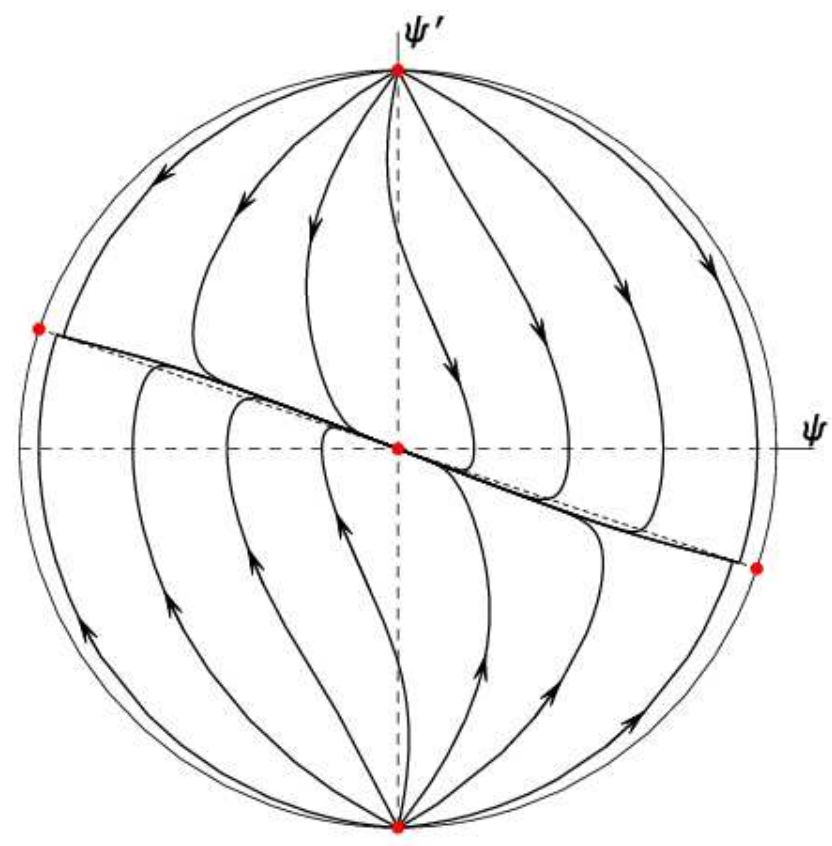

d) c)

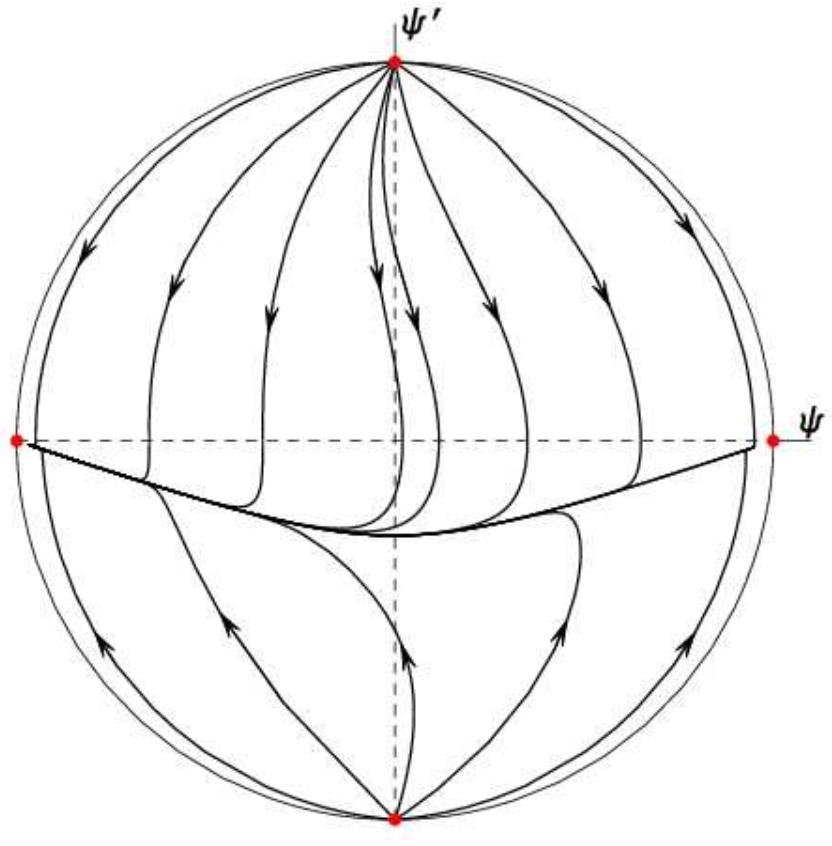

FIG. 4: Phase portraits with compactification at infinity for minimally coupled phantom scalar field $\xi=0$ for various potential functions. a) $U(\psi) \propto \psi^{2}$, critical point at $\psi^{\prime}=0$ and $\psi=\infty$ plays the role of global attractor, $w_{X}=-1$ at this point; b) $U(\psi) \propto \psi^{-\alpha}$ and $\alpha=1$; c) $U(\psi) \propto \exp (-\lambda \psi)$ and $\lambda=1$, critical point at $\psi^{\prime}=0$ and $\psi=-\infty$ is the global attractor of the dynamical system and $w_{X}=-1$ at this point; critical point at $\psi^{\prime}=0, \psi=\infty$ is a saddle type; d) $U(\psi) \propto \exp \left(-\lambda \psi^{2}\right)$ and $\lambda=1, \xi=0$, the critical point at finite domain at $\psi^{\prime}=\psi=0$ plays the role of global attractor and $w_{X}=-1$ at this point, the points at infinity $\psi^{\prime} \neq 0, \psi \neq 0$ are a saddle type.

paths of the extended quintessence in the phase space for all admissible initial conditions. The structure of the phase space crucially depends on the constant of the non-minimal coupling because right-hand sides of the dynamical system depends on this parameter. We investigate bifurcations under changing of this parameter to distinguish some generic evolutional routes to the FRW model with the cosmological constant. We found that dynamical system admits the invariant submanifold $\left(\psi, \psi^{\prime}\right)$ and trajectories in the long time evolution approach to this submanifold of the system. The behavior of trajectories on this phase plane give us information how the FRW model with the cosmological constant appears as a final asymptotic state - a limit set. Our main conclusion is that there are principally two 

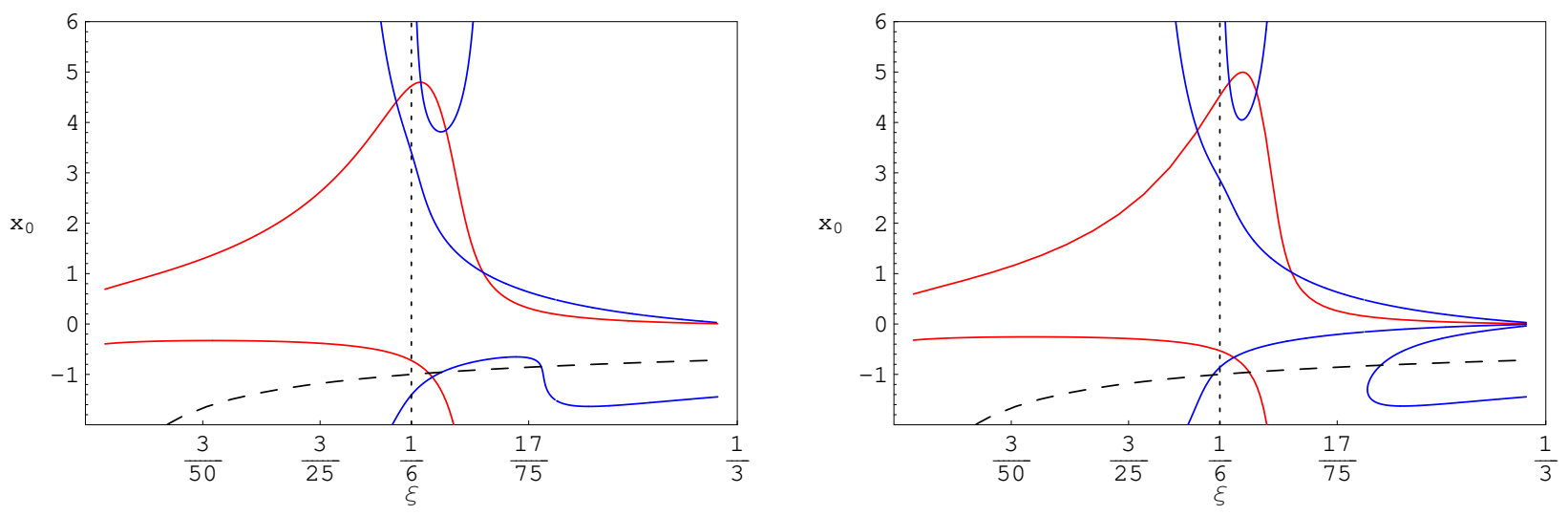

FIG. 5: Dependence of $x_{0}$ on the coupling constant $\xi$ for two first terms in the Taylor series of the equation of state parameters (27) and (35): light gray (red el.version) - first term, dark gray (blue el.version) - second term and two linear parametrizations in the scale factor (left panel) and in the redshift (right panel), values of $w_{0}$ and $w_{1}$ from [34]. The horizontal dashed line denotes unphysical limit on the values of $x_{0}<-\frac{1}{\sqrt{6 \xi}}$. It is interesting that astronomical data together with assumption $y_{0}=-\frac{3}{2} x_{0}$ indicate the value of $\xi$ parameter near conformal coupling $\xi=\frac{1}{6}$.

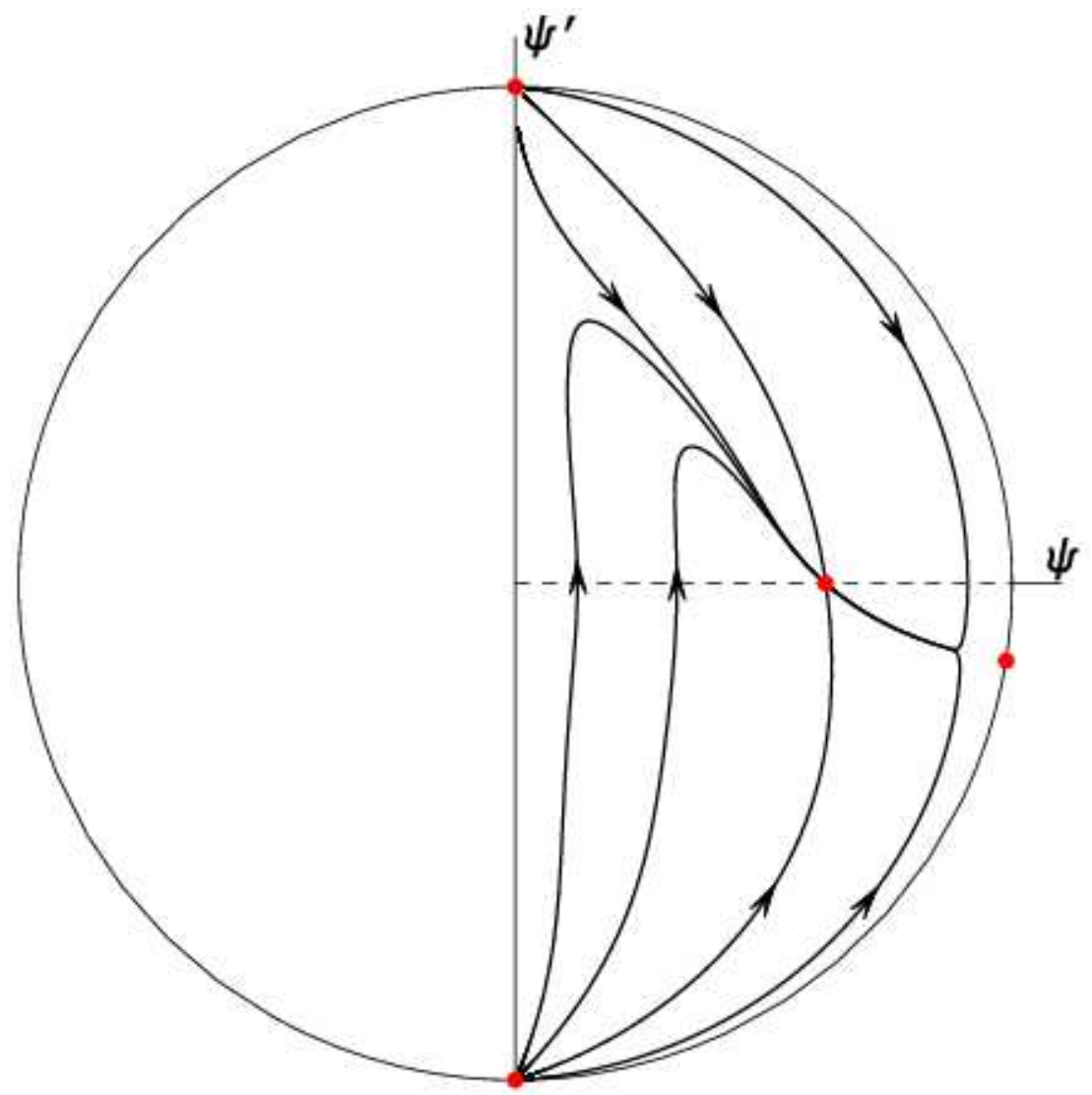

FIG. 6: The phase portrait with compactification at infinity for $\xi=3 / 50$. The critical point at infinity is a saddle type.

generic evolutional scenarios of approaching the limit set in the future:

1. node type scenario,

2. focus type scenario (repellor-attractor scenario).

In the first type of scenario trajectories approach the critical point monotonically without oscillations. In the second type of scenario the approach to the final state is through the characteristic infinite number of damping quasi- 


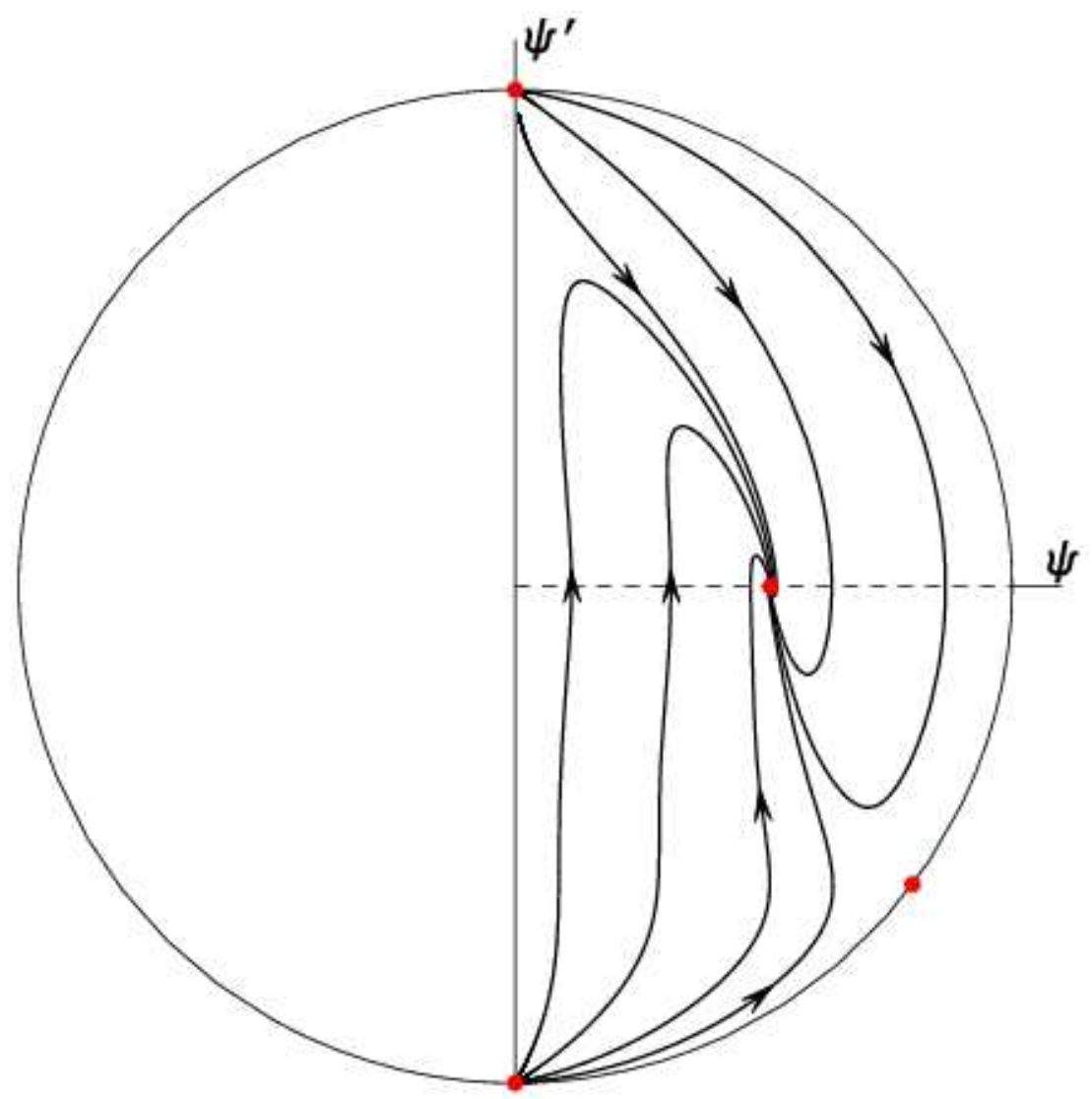

FIG. 7: The phase portrait with compactification at infinity for $\xi=3 / 20$. The critical point of a focus type plays the role of a global attractor in the phase space.

oscillations. Additionally in this case the final limit set is always a focus type and it is achieved by evolution from an unstable node.

It is shown that the type of evolutional scenario describing the approach toward the stable limit set in the future crucially depends on the trace and the determinant of the linearization matrix of the system on the invariant submanifold. They can be simply expressed in the terms of slow-rolling parameters, i.e., in terms of geometry of a potential function of the phantom scalar field. It was also demonstrated how our conclusion depends on the choice of a special form of a potential function of the scalar field. We found that in the generic case two mentioned before scenarios are typical. We obtained the exact form of coefficient of the equation of state $w(z)$ near the present epoch directly from the dynamics of the model. This gave us the form of $w(z)$ which can be used in estimation of this parameter. Such a methodology is more appropriate in our belief than taking the ad-hoc form of $w(z)$ usually in a linear form.

\section{Acknowledgments}

This work has been supported by the Marie Curie Actions Transfer of Knowledge project COCOS (contract MTKDCT-2004-517186).

[1] N. A. Chernikov and E. A. Tagirov, Annales Poincare Phys. Theor. A9, 109 (1968).

[2] C. G. Callan Jr., S. R. Coleman, and R. Jackiw, Ann. Phys. 59, 42 (1970).

[3] N. D. Birrell and P. C. W. Davies, Quantum Fields in Curved Space (Cambridge University Press, Cambridge, 1984).

[4] V. Faraoni, Int. J. Theor. Phys. 40, 2259 (2001), arXiv:hep-th/0009053.

[5] R. M. Wald, General Relativity (The University of Chicago Press, Chicago, 1984).

[6] V. Faraoni, Phys. Rev. D53, 6813 (1996), arXiv:astro-ph/9602111. 




FIG. 8: The phase portrait with compactification at infinity for $\xi=17 / 75$. The focus type critical point is no longer a global attractor in the phase space.

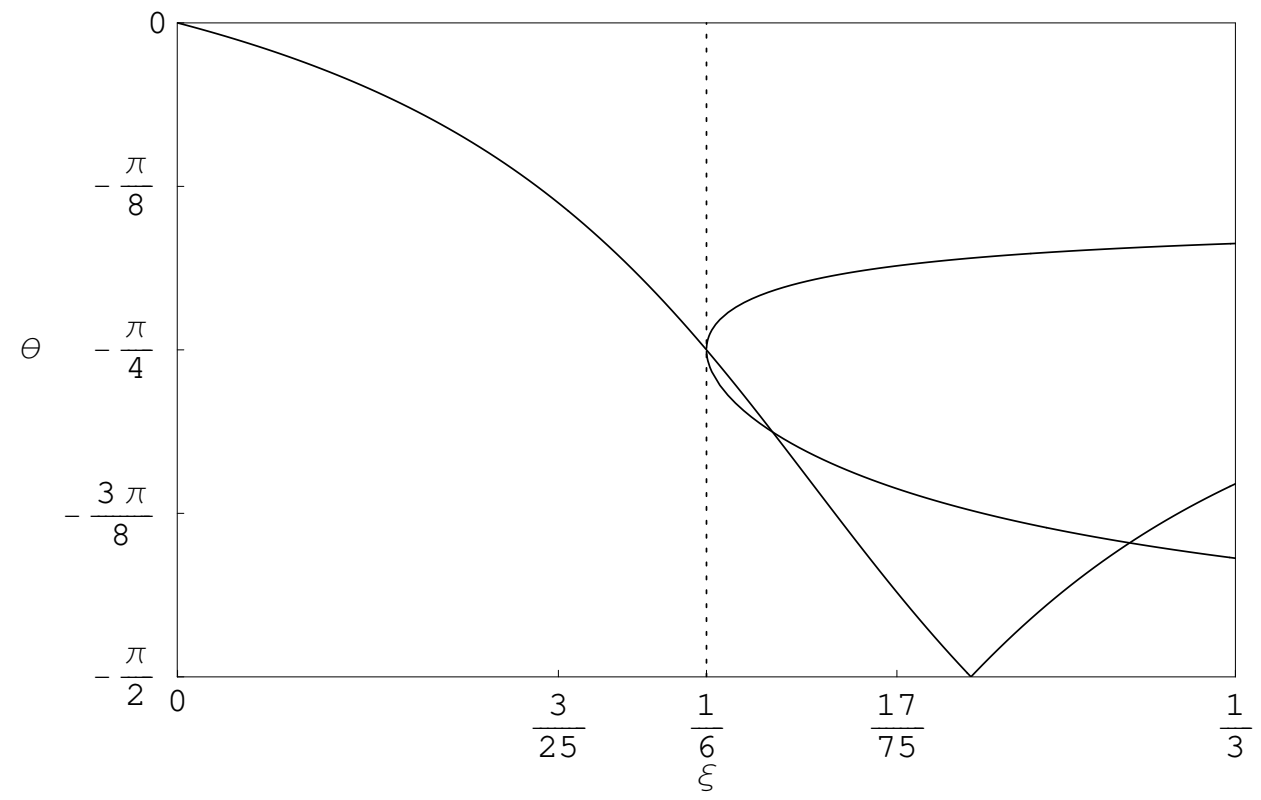

FIG. 9: The localization and number of critical points at infinity as a function of coupling constant $\xi$. 
[7] S. Sonego and V. Faraoni, Class. Quant. Grav. 10, 1185 (1993).

[8] A. P. Lightman, W. H. Press, R. H. Pirce, and S. A. Teukolsky, Problem Book in Relativity and Gravitation (Princeton University Press, Princeton, 1975).

[9] V. Faraoni, Does the non-minimal coupling of the scalar field improve or destroy inflation? (1997), arXiv:gr-qc/9807066.

[10] S. Tsujikawa and B. Gumjudpai, Phys. Rev. D69, 123523 (2004), arXiv:astro-ph/0402185.

[11] A. G. Riess, A. V. Filippenko, P. Challis, A. Clocchiattia, A. Diercks, P. M. Garnavich, R. L. Gilliland, C. J. Hogan, S. Jha, R. P. Kirshner, et al. (Supernova Search Team), Astron. J. 116, 1009 (1998), arXiv:astro-ph/9805201.

[12] P. Astier, J. Guy, N. Regnault, R. Pain, E. Aubourg, D. Balam, S. Basa, R. Carlberg, S. Fabbro, D. Fouchez, et al. (The SNLS Collaboration), Astron. Astrophys. 447, 31 (2006), arXiv:astro-ph/0510447.

[13] T. M. Davis, E. Mortsell, J. Sollerman, A. C. Becker, S. Blondin, P. Challis, A. Clocchiatti, A. V. Filippenko, R. J. Foley, P. M. Garnavich, et al., Astrophys. J. 666, 716 (2007), arXiv:astro-ph/0701510.

[14] D. N. Spergel, R. Bean, O. Dor, M. R. Nolta, C. L. Bennett, J. Dunkley, G. Hinshaw, N. Jarosik, E. Komatsu, L. Page, et al. (WMAP Collaboration), Astrophys. J. Suppl. 170, 377 (2007), arXiv:astro-ph/0603449.

[15] I. Zlatev, L.-M. Wang, and P. J. Steinhardt, Phys. Rev. Lett. 82, 896 (1999), arXiv:astro-ph/9807002.

[16] S. M. Carroll, Phys. Rev. Lett. 81, 3067 (1998), arXiv:astro-ph/9806099.

[17] S. Bludman, What we already know about quintessence (2003), arXiv:astro-ph/0312450.

[18] B. Ratra and P. J. E. Peebles, Phys. Rev. D37, 3406 (1988).

[19] C. Wetterich, Nucl. Phys. B302, 668 (1988).

[20] V. Faraoni, Phys. Rev. D62, 023504 (2000), arXiv:gr-qc/0002091.

[21] J.-P. Uzan, Phys. Rev. D59, 123510 (1999), arXiv:gr-qc/9903004.

[22] L. Amendola, Phys. Rev. D60, 043501 (1999), arXiv:astro-ph/9904120.

[23] T. R. Choudhury and T. Padmanabhan, Astron. Astrophys. 429, 807 (2005), arXiv:astro-ph/0311622.

[24] V. Faraoni and M. N. Jensen, Class. Quant. Grav. 23, 3005 (2006), arXiv:gr-qc/0602097.

[25] R. R. Caldwell, Phys. Lett. B545, 23 (2002), arXiv:astro-ph/9908168.

[26] M. P. Dabrowski, T. Stachowiak, and M. Szydlowski, Phys. Rev. D68, 103519 (2003), arXiv:hep-th/0307128.

[27] V. Faraoni, Cosmology in Scalar-Tensor Gravity, vol. 139 of Fundamental Theories of Physics (Kluwer Academic Publishers, Dordrecht, 2004).

[28] Y. Fujii and K. Maeda, The Scalar-Tensor Theory of Gravitation (Cambridge University Press, Cambridge, 2003).

[29] A. Fuzfa and J. M. Alimi, Phys. Rev. D75, 123007 (2007), arXiv:astro-ph/0702478.

[30] E. Gunzig, A. Saa, L. Brenig, V. Faraoni, T. R. Filho, and A. Figueiredo, Phys. Rev. D63, 067301 (2001), arXiv:grqc/0012085.

[31] E. Gunzig, V. Faraoni, A. Figueiredo, T. M. Rocha, and L. Brenig, Class. Quant. Grav. 17, 1783 (2000).

[32] O. Hrycyna and M. Szydlowski, Phys. Lett. B651, 8 (2007), arXiv:0704.1651 [hep-th].

[33] M. Szydlowski, O. Hrycyna, and A. Krawiec, JCAP 0706, 010 (2007), arXiv:hep-th/0608219.

[34] D. A. Dicus and W. W. Repko, Phys. Rev. D70, 083527 (2004), arXiv:astro-ph/0407094.

[35] L. Perko, Differential Equations and Dynamical Systems (Springer-Verlag, New York, 1991). 